\title{
British docs urge elective ventilation
}

$\mathrm{S}$ ome might call it harvesting organs. But the British Medical Association (BMA) says the demand and need for transplantable organs is so acute that the time has come for Britain to consider keeping nearly brain-dead patients alive solely for the purpose of culling their hearts, livers and kidneys, even if it means that a few ethical compromises have to be made.

It's called "elective ventilation," in which comatose patients close to death are placed on ventilation for the sole purpose of culling their organs once brainstem death occurs. It's a practice that's already common in the United States and Spain but was banned by the United Kingdom's Department of Health in 1994.

Yet, with more than 1000 people dying annually in the UK while on a transplant waiting list that typically has about 8000 people in need of organs, the time has come for a public debate on whether the UK should get into the business of harvesting organs, BMA argued in a report, Building on Progress: Where next for organ donation policy in the UK? (http://bma.org .uk/-/media/Files/PDFs/Working\%20for $\%$ 20change/Improving\%20health/organ donation_buildingonprogressfebruary 2012.pdf).

Elective ventilation is among a series of measures that the association says must be evaluated to bolster organ availability. Others included broadening the donor pool by allowing for organs to be culled from so-called higher-risk patients, such as the elderly or those with a malignancy; allowing removal of a heart that has been restarted after the patient died following a cardiorespiratory arrest; introducing presumed consent for organ donation, unless a dying patient has indicated his objections; giving registered organ donors priority status on waiting lists in the event they need an organ; and using the hearts of babies who are less than three months

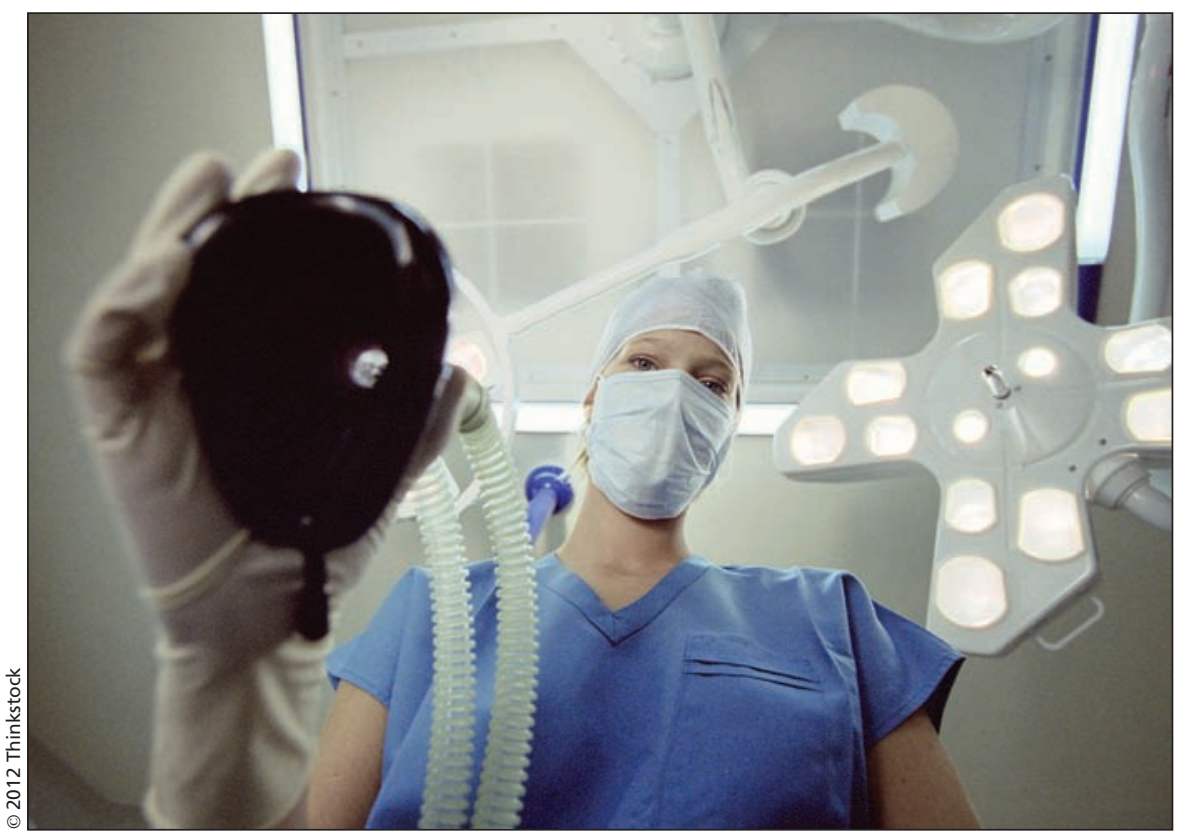

Elective ventilation prior to organ donation is already common in the United States and Spain.

old but who've been given no chance of survival.

The introduction of such measures, including elective ventilation, must be given serious consideration if organ supply is to ever meet demand, BMA argued. "As a society we now need to consider whether, having developed the infrastructure, we should be satisfied that we have done all we can or whether we should seek to build on what has already been achieved by shifting our attention to additional ways of increasing the number of organ donors.'

"Elective ventilation is not an easy option but it has been shown to increase donation rates, and to facilitate the wishes of a group of patients who want to donate and would otherwise be unable to do so," the report states, adding that "the BMA is not calling for the law to be changed to permit elective ventilation but believes this may be an issue that would benefit from debate both to assess the clinical, legal and ethical issues raised and to assess public opinion about its use.'

There is some evidence that sup- ports the proposition that elective ventilation increases the supply of organs. There was a $50 \%$ increase in the number of transplantable organs at the Royal Devon \& Exeter Hospital in Exeter, England, following the institution of a protocol during the 1980s for transferring patients to an intensive care unit to be ventilated until their organs could be recovered, one study indicated (Lancet 1990; 335: 1133-5).

But the UK National Health Service ordered the protocol to be abandoned in 1994 on the grounds that it was unlawful to ventilate a patient for the purpose of harvesting his or her organs as it did not constitute a procedure that was being undertaken for his or her benefit, particularly without his or her consent.

Yet the need for organs is so great that a public debate on the issue has to be held, says Dr. Vivienne Nathanson, director of professional activities for the BMA. "We're not saying it's the right thing, but we're also saying it's such a big change in the way we regard ... the integrity of the body that we need a public debate before we 
decide whether it's something we should do or not."

Although the proposition has largely been met by silence from politicians and other health groups, Nathanson says the association hopes the issue will be taken up when the UK Organ Donation Taskforce issues a report in 2013 identifying barriers to organ donation and recommending measures to increase donor rates.

Still at issue is whether the same legal environment exists in Britain today, as existed when elective ventilation was outlawed in 1994, particularly surrounding the issue of consent. According to one study, the introduction of the Mental Capacity Act 2005 changed the legal picture (BMJ 2008; 336: 1346-7). A provision of the act stipulates that in cases where a patient is incapable of providing consent for a medical procedure, decision-makers must consider his "past and present wishes and feelings ... the beliefs and values that would be likely to influence his decision if he had capacity, and the other factors that he would be likely to consider if he were able to do so" (www.legislation.gov.uk/ukpga/2005/9 /section/4).

It raises some tricky possibilities, notes John Coggon, a research fellow at the Institute for Science, Ethics and Innovation in the School of Law at the University of Manchester in the UK. Elective ventilation includes a small risk that a patient's condition might not progress to brainstem death as expected, potentially leaving him in a persistent vegetative state - wherein he is able to breathe on his own but has no evidence of higher-brain activity — or a similar condition, Coggon writes in an email. Whether that risk means the procedure could be judged not to be in a patient's best interest despite an expressed desire to be an organ donor or consent from relatives "is an open question."

Permitting elective ventilation would present ethical and logistical challenges, BMA noted in its report. For one, maintaining mechanical ventilation would require resources in the form of space in intensive care units. "In the BMA's view, priority would always need to be given to the use of intensive care facilities for those who have a chance of recovery rather than for those who are being ventilated to facilitate donation."

As for the possibility that a patient might survive in a persistent vegetative state, "very careful safeguards" would have to be implemented to reduce that risk, including "restricting elective ventilation to those patients dying of spontaneous intracranial haemorrhage (since these patients rarely, if ever, develop pvs [persistent vegetative state]) and stating that artificial ventilation must not be started until natural respiratory arrest has occurred," the report stated. - Michael Monette, CMAJ

CMAJ 2012. DOI:10.1503/cmaj.109-4259

Editor's note: First of a three-part series.

Next: The ever-muddled Canadian waters and elective ventilation 